\title{
Numerical and analytical investigation of the influence of porosity on the frequency response of GLARE composite
}

\author{
Istvan A. Veres \\ Department of Mechanical Engineering \\ University Walk, University of Bristol \\ Bristol BS8 1TR, UK \\ RECENDT GmbH, Altenberger Str. 69 \\ 4040 Linz, Austria \\ Email: istvan.veres@recendt.at
}

\author{
Robert A. Smith \\ Department of Mechanical Engineering \\ University Walk, University of Bristol \\ Bristol BS8 1TR, UK
}

\author{
Valerie J Pinfield \\ Chemical Engineering Dept. \\ Loughborough University \\ Loughborough, Leics., UK
}

\begin{abstract}
In this study, the validity of the effective-medium model approach to model the ultrasonic response of porosity is investigated with the help of time-domain Finite Element Method simulations. The effective-medium model is based on a single scattering approach i.e. by neglecting the rescattering of the waves and assuming a complex wave number to account for the frequency-dependent attenuation of the elastic waves. The numerical model, on the other hand, allows the study of the scattering of the elastic waves on randomly distributed spherical cavities and also accounts for the multiple scattering effects.
\end{abstract}

\section{INTRODUCTION}

Fibre-metal laminates such as GLARE (Glass Laminate Aluminium Reinforced Epoxy) provide enhanced fatigue resistance compared with unreinforced metals. The composite layers, however, frequently suffer from air inclusions, or porosity, which reduces the overall strength and performance of the laminate. During the propagation of ultrasonic waves through such a porous material the waves are scattered at the randomly distributed voids. This scattering leads to a frequency-dependent attenuation as previously demonstrated [1]. Measurement of this attenuation allows the evaluation of the bulk-averaged porosity. The measurement principle is in general independent of the investigated material and has been successfully applied in composites [2] and in aluminum castings [3], [4], [5] but does not provide any information about the depth distribution of the porosity. For this, a pulseecho method is required but the reflected waveform is complex for GLARE due to the high reflection coefficients at each interface. Small changes in the glass-fibre layer thicknesses cause large changes in the reflected response, making it impossible to distinguish signals from porosity.

In the applied effective-medium model a complex wave number is used to match the response of a homogenous medium to the frequency-dependent response of a random distribution of cavities, calculated from the scattering from a single cavity [7], [8], [9]. As well as effective-medium models, also numerical simulation can be used to study wave-propagation problems in inhomogeneous media, such as periodic structures [10] or random media [11]. In the presented work, both techniques are applied to model porosity. In particular, three-dimensional time-domain FEM simulations are carried out to model the scattering of the elastic waves on randomly distributed cavities. The range of validity of the effective-medium model is investigated by comparing calculated transmission spectra for various porosity levels and distributions to the numerical simulations.

\section{ANALYTICAL AND NUMERICAL MODELING OF WAVE PROPAGATION IN RANDOM MEDIUM}

Within an effective-medium model the ultrasonic scattering from porosity is taken into account by using a complex wave number. The 3D time-domain FEM simulations, on the other hand, are carried out by modeling the scattering of the ultrasonic waves at a random arrangement of spherical voids. The results of the two models are compared to verify and to investigate the limitations of the frequency-domain effectivemedium model.

\section{A. Effective-medium model}

For a particular volume fraction $\phi$ of cavities the complex wave number $K$ of the effective medium [7], [8] is given as:

$$
K^{2}=k^{2}+\frac{3 \phi}{R^{3}} f(0),
$$

where $k$ is the real wave number and $f(0)$ is the backscattered amplitude. For simplicity it is assumed that the pores are spherical scatterers with radius $R$. For longitudinal waves the backscattered amplitude can be obtained as [8]:

$$
f(0)=\frac{1}{i k} \sum_{n=0}^{\infty}(2 n+1) A_{n},
$$

with $A_{n}$ being the scattering coefficients of order $n$ for a spherical cavity. For transverse waves, the definition of the scattering cross-section in Eq.(2) is slightly different due to the 
(a)

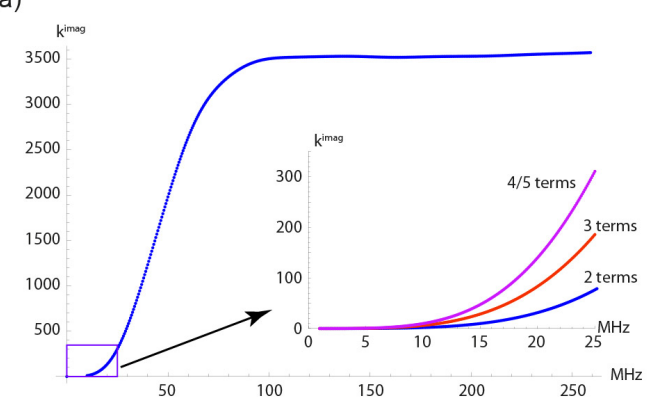

(b)

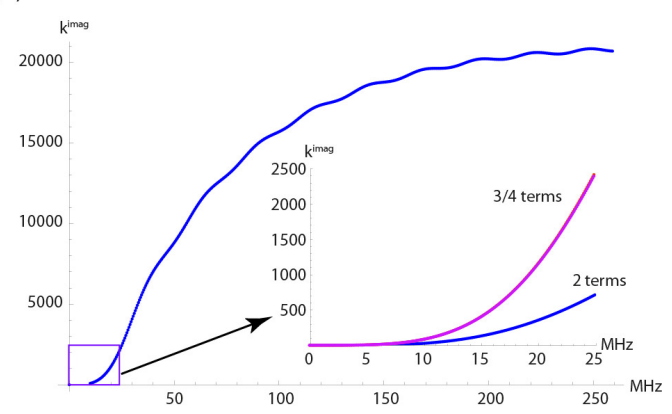

Fig. 1. Attenuation coefficients from cavities with radius $R=10 \mu \mathrm{m}$ and volume fraction $\phi=5 \%$ with $n=15$ terms. (a) Longitudinal waves and (b) shear waves. The insets show the convergence of the attenuation coefficients with up to 5 terms.

presence of two transverse waves with different polarizations [13]:

$$
f(0)=\frac{1}{i k} \sum_{n=1}^{\infty}(2 n+1)\left(B_{n}+C_{n}\right),
$$

where $B_{n}, C_{n}$ are the scattering coefficients for the transverse waves. The scattering of plane waves from spherical obstacles, such as cavities or inhomogeneities, is a well-known problem. The scattering coefficients $\left(A_{n}, B_{n}, C_{n}\right)$ can be found in the literature for longitudinal waves [14] and also for transverse waves [13]. The infinite sum in Eq.(2) can be truncated; in the current work 15 terms were used, which provides sufficient accuracy up to $250 \mathrm{MHz}$ for the current system. Calculated attenuation coefficients for cavities with radius $R=10 \mu \mathrm{m}$, volume fraction $\phi=5 \%$, and longitudinal $\left(c_{l}\right)$ and shear $\left(c_{s}\right)$ wave velocities of $c_{l}=3330 \mathrm{~ms}^{-1}$ and $c_{s}=1870 \mathrm{~ms}^{-1}$ are shown in Fig.1 with $n=15$ terms. The insets of the Figure show the convergence for a low-frequency range, below $\sim 25 \mathrm{MHz}$ where approximately 5 terms are sufficient.

Cavities of unequal radii, i.e. with a distribution of radii, can be evaluated by using an effective attenuation $\alpha_{\text {eff }}$ [5]. For the corresponding average void volume fraction of $\bar{\phi}$ the effective attenuation is the sum of the imaginary parts of the effective wave numbers for each of the $i$ th pore sizes within a discretised distribution:

$$
\alpha_{e f f}=\sum_{i} K_{i}^{i m a g}
$$

where the complex wave numbers $K_{i}$ are calculated for the $i$ th discrete range of radii (with $R_{i}$ ) and the corresponding volume fractions $\phi_{i}$ is given as:

$$
\phi_{i}=\bar{\phi} \frac{R_{i}^{3}}{\sum_{i} R_{i}^{3}} .
$$

These relatively simple relationships are used to assign a complex wave number to the propagating waves. The influence of the porosity on the ultrasound propagation is investigated in the following by using this model, and compared to 3D time-domain FEM simulations.

\section{B. Wave propagation through a porous layer}

The scattering of the ultrasonic waves at a random distribution of cavities is simulated using a time-domain FEM model. For this purpose the commercial FEM package PZFlex (Weidlinger A., Inc.) was used. The applied model is shown in Fig. 2. The FEM model consists of two aluminium layers with $400 \mu \mathrm{m}$ thicknesses each and a porous composite layer with $250 \mu \mathrm{m}$ thickness whereby the pores are modeled as randomly-distributed spherical voids. The spherical voids were arranged around the centre of a cell in a 3D cuboid lattice 3D cuboid lattice $(87.5 \mu \mathrm{m} \times 87.5 \mu \mathrm{m} \times 50 \mu \mathrm{m})$. The distance from the center of the cuboid cell was randomly chosen within twice of the lattice constant in each direction. In the $y, z$ directions symmetric boundary conditions (BCs) were used; along the $x$ direction, at both ends, absorbing BCs were applied. The time-domain results of the 3D FEM simulations were converted into frequency-domain responses by evaluating the frequency-dependent attenuation of the waves, represented by the transmitted spectrum. This is demonstrated in Fig. 3(a) showing the normalized input and transmitted frequencies from an incident plane wave generated along the $x$ direction with a Gaussian envelope with a full-width at half maximum of $20 \mathrm{~ns}$. The transmission spectrum is obtained by dividing

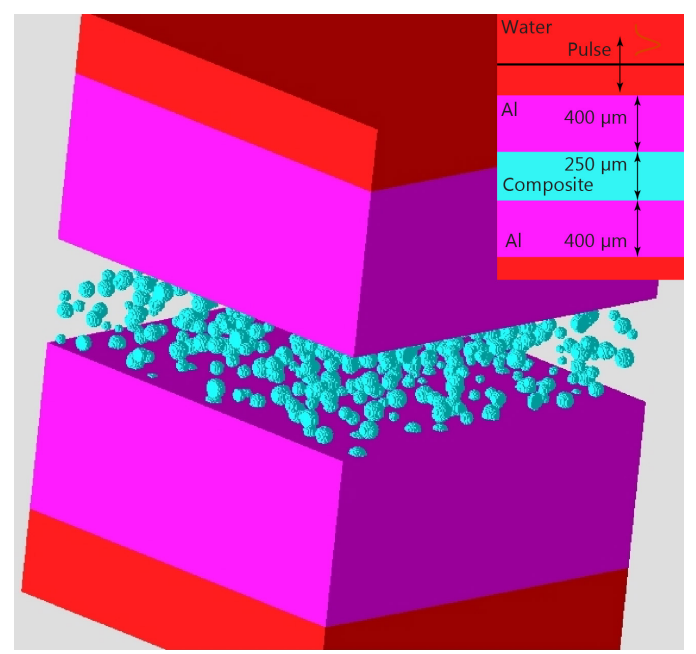

Fig. 2. 3D FEM model of the scattering problem. The model is consisting of two aluminum layer and a porous composite layer. For improved visibility of the pores, the image shows the pores as red inclusions in a transparent material; in the simulations the shown spherical particles are voids in the composite material. 
(a)

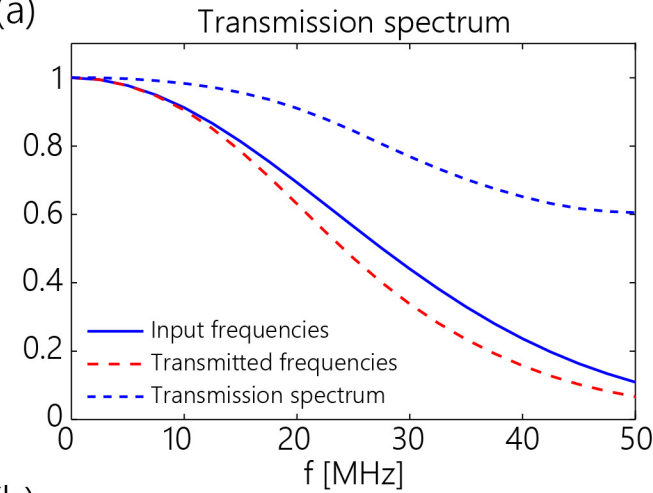

(b)

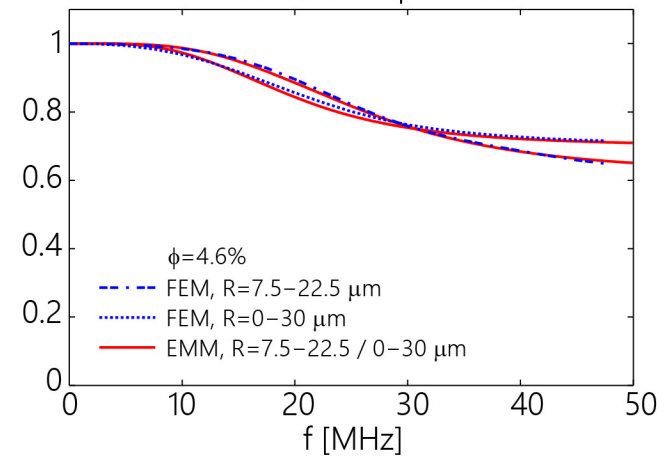

Fig. 3. (a) Input and transmitted time-domain pulses for the FEM. (b) Transmission spectra from two FEM simulations with identical volume fractions but different scatterer diameter distributions, compared to the effective-medium model (EMM).

the transmitted spectrum by the input spectrum. Since the description of the random scattering problem requires the determination of mean values of the wave propagation, the captured responses were averaged across the cross-section of the models in the $y-z$ plane (Fig.2). The transmission spectra from the effective medium model was calculated by inserting the complex wave number $K^{i m a g}$ into a harmonic function $\left(e^{-\Delta x K^{i m a g}}\right)$ with $\Delta x=250 \mu \mathrm{m}$.

\section{RESULTS AND DISCUSSION}

In the FEM simulation the modeled cross section was $0.975 \mathrm{~mm} \times 0.975 \mathrm{~mm}$ with element dimensions of $\Delta x=2 \mu \mathrm{m}$ and $\Delta y=\Delta z=2.6 \mu \mathrm{m}$. The total number of elements was $181 \times 10^{6}$ (corresponding to a discretisation with $542 \times 10^{6}$ DOF). Within the composite layer 726 spherical scatterers with a radius of $16 \mu \mathrm{m}$ were randomly distributed whereby the total volume fraction of the pores was $4.7 \%$. To verify the FEM model, simulations with different geometrical configurations were performed and the averaged transmission spectra compared. ${ }^{1}$ The comparison has shown, that the averaged response of the model is independent of the geometrical

\footnotetext{
${ }^{1}$ Three simulations were performed, each with 726 randomly arranged spherical scatterer with radii $R=16 \mu \mathrm{m}$. The arrangements were different in all cases. The intersection of the spheres was allowed leading to the total volume fraction of $4.662 \%, 4.403 \%$ and $4.537 \%$ respectively. The models consisted of $180.6 \times 10^{6}$ elements corresponding to $541.8 \times 10^{6} \mathrm{DOF}$.
}

configuration.

In further simulations the effect of varying the pore diameter was studied. In these simulations two ranges of pore diameters were used $R=7.5-22.5 \mu \mathrm{m}$ and $R=0-30 \mu \mathrm{m}$ both with a uniform distribution of the diameters and with the same total void volume fraction. In the first case $(R=7.5-22.5 \mu \mathrm{m})$ 726 , in the second case $(R=0-30 \mu \mathrm{m}) 486$ spheres were randomly arranged leading to total volume fractions of $4.62 \%$ and $4.59 \%$ respectively. Similarly to the previous simulations the intersection of the spheres was allowed and the models consisted of $181 \times 10^{6}$ elements corresponding to $542 \times 10^{6}$ DOF. The attenuation coefficients for the effective-medium model were calculated by using Eq.4 and a comparison with the FEM models in Fig. 3(b) shows that the effective-medium model is a good approximation of the FEM solution. In addition, by comparing the curves, it is clearly-visible that the broader void-size distribution leads to a stronger attenuation for lower frequencies but the curve flattens out with a higher transmission coefficient. This property might allow a unique determination of the volume fraction or the void-size distribution from an inverse problem if one of these properties is known and measurement data is available for sufficiently high frequencies.

Next, different cavity diameters and different volume fractions were studied. Three simulations were performed, each with 726 randomly arranged spherical scatterer with radii $R=10 / 16 / 20 \mu \mathrm{m}$. The geometric arrangements remained identical in all cases - i.e. the location of the pores remained the same only the diameter was increased - leading to total volume fractions of $1.1 \%, 4.7 \%$ and $9.4 \%$ respectively. Similarly to the previous simulations the intersection of the spheres was allowed and the models consisted of $180.6 \times 10^{6}$ elements corresponding to $541.8 \times 10^{6}$ DOF.

The transmission spectra in Fig. 4(a) of the three systems with radii $R=10 / 16 / 20 \mu \mathrm{m}$ were evaluated from the FEM simulation similarly to the previous cases using the spatially averaged time-domain responses. The comparison with the effective-medium model shows that this model is a good approximation of the numerical simulation for the cases with radii $R=10 / 16 \mu \mathrm{m}$ (total volume fractions of $1.1 \%, 4.7 \%$ ). For the largest diameter $R=20 \mu \mathrm{m}$ with a total volume fraction of $9.4 \%$ deviations starts to become visible as the effective medium-model underestimates the attenuation. Finally, a simulation with high void volume fraction $(\phi=25.86 \%, R=16 \mu \mathrm{m})$ was also carried out and compared to the prediction of the effective mediummodel. The transmission spectrum in Fig. 4(b) shows that the approximation using a complex wave number and neglecting multiple scattering leads to a significant error.

\section{CONCLUSion}

In the presented paper an effective-medium model was compared to a 3D FEM numerical model. Calculations were carried out by assuming identical spherical scatterers with diameters up to $60 \mu \mathrm{m}$ and by assuming different size distributions. The comparison of the models included different pore 
(a)

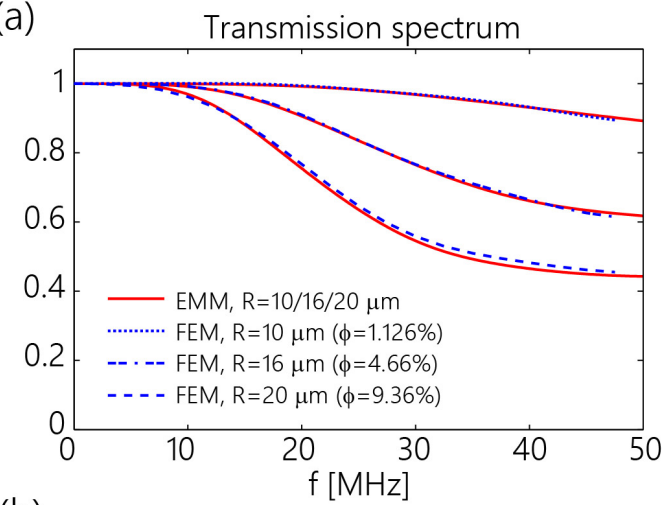

(b)

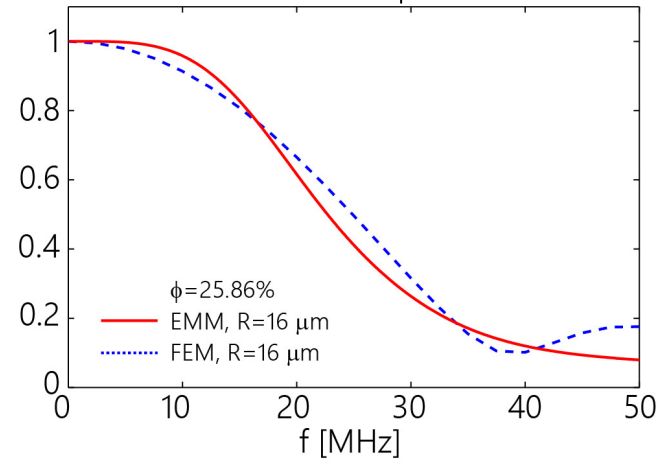

Fig. 4. (a) Analytical and numerical transmission spectra for three different scatterer diameters and volume fractions. The effective medium model shows a good agreement with the FEM simulations. (b) Transmission spectra from a simulation with large volume fraction (25.9\%) compared to the transmission spectrum of the effective medium-model. In this case the effective medium model fails to predict the transmission spectrum.

diameters, volume fractions and pore-diameter distributions. The transmissibility of the porous layer was evaluated from simulations and the results compared to the prediction of the analytical model.

The calculated transmission spectra from the two models show a good agreement for porosity values below $10 \%$. Hence, the frequency-dependent attenuation of the ultrasonic waves is well-estimated by the single-scattering approximation of the effective medium model for reasonable levels of volume fractions - below and around $10 \%$.

\section{ACKNOWLEDGMENT}

Support is acknowledged from an ongoing research program of the UK Engineering and Physical Sciences Research Council EPSRC (EP/K037315/1). The Authors gratefully acknowledge the help of M. Caleap, B. Drinkwater and P.D. Wilcox for valuable discussions.

\section{REFERENCES}

[1] J. Gubernatis and E. Domany, "Effects of microstructure on the speed and attenuation of elastic waves in porous materials," Wave Motion, vol. 6 , no. 6, pp. $579-589,1984$.

[2] D. Stone and B. Clarke, "Ultrasonic attenuation as a measure of void content in carbon-fibre reinforced plastics," Non-Destructive Testing, vol. 8, no. 3, pp. $137-145,1975$.

[3] L. Adler, J. H. Rose, and C. Mobley, "Ultrasonic method to determine gas porosity in aluminum alloy castings: Theory and experiment," $J$. Appl. Phys., vol. 59, no. 2, 1986.

[4] S. Nair, D. Hsu, and J. Rose, "Porosity estimation using the frequency dependence of the ultrasonic attenuation," J. Nondestruct. Eval., vol. 8 , no. 1 , pp. 13-26, 1989.

[5] B. Ghaffari, G. Mozurkewich, L. A. Godlewski, and J. W. Zindel, "Ultrasonic characterization of shrinkage microporosity in aluminum castings," Ultrasonics, vol. 41, no. 9, pp. 699 - 707, 2004.

[6] V. K. Varadan, Y. Ma, and V. V. Varadan, "A multiple scattering theory for elastic wave propagation in discrete random media," J. Acoust. Soc. Am., vol. 77, no. 2, 1985.

[7] V. J. Pinfield, R. E. Challis, and R. A. Smith, "A comparison of stochastic and effective medium approaches to the backscattered signal from a porous layer in a solid matrix," J. Acoust. Soc. Am., vol. 130, no. 1, 2011.

[8] V. J. Pinfield and R. E. Challis, "Simulation of incoherent and coherent backscattered wave fields from cavities in a solid matrix," J. Acoust. Soc. Am., vol. 132, no. 6, 2012.

[9] V. J. Pinfield and R. E. Challis, "Modelling the backscatter from spherical cavities in a solid matrix: Can an effective medium layer model mimic the scattering response?" J. Phys.: Conf. Ser., vol. 269, no. 1, p. 012016.

[10] I. A. Veres, D. M. Profunser, A. A. Maznev, A. G. Every, O. Matsuda, and O. B. Wright, "Point source in a phononic grating: stop bands give rise to phonon-focusing caustics," N. J. Phys., vol. 14, no. 12, p. 123015 , 2012.

[11] M. Muller, E. Bossy, F. Meziere, and A. Derode, "2d numerical simulations of ultrasound propagation in random anisotropic media: Occurrence of two longitudinal waves in bone-like structures," in $\mathrm{Ul}$ trasonics Symposium (IUS), 2011 IEEE International, Oct 2011, pp. 1614-1617.

[12] L. L. Foldy, "The multiple scattering of waves. i. general theory of isotropic scattering by randomly distributed scatterers," Phys. Rev., vol. 67, pp. 107-119, Feb 1945.

[13] N. G. Einspruch, E. J. Witterholt, and R. Truell, "Scattering of a plane transverse wave by a spherical obstacle in an elastic medium," Journal of Applied Physics, vol. 31, no. 5, 1960.

[14] K. Graff, Wave motion in elastic solids. Mineola, New York: Dover Publications Inc., 1991 